\title{
Presentación. \\ El estudio de la cerámica prehistórica, una investigación global
}

\author{
Xavier Clop García ${ }^{1}$, Jaume García Rosselló ${ }^{2}$
}

En los últimos años, se está desarrollando un interés renovado por el desarrollo de estudios en torno a la cerámica prehistórica. Este interés renovado se ve reflejado en la aparición de publicaciones ambiciosas, que en algunos casos plantean cuestiones referentes a todos los aspectos vinculados a la investigación de un conjunto cerámico, en otros abordan de manera extensa cómo realizar el estudio de un aspecto específico de los productos cerámicos y en otros realizan aportaciones a partir del estudio de comunidades actuales que tienen estructuras de producción de la cerámica que se consideran similares a las que se pudieron desarrollar durante la prehistoria reciente (p. ej. García Rosselló y Calvo Trías, 2013; Albero, 2014; Roux, 2016; Hunt, 2017; Burnez-Lanotte, 2017; Alarcón García et al., 2018).

Esta situación pasa por la ampliación de las posibilidades de estudio de los productos cerámicos, más allá de los imprescindibles estudios morfotipológicos y de las técnicas y motivos decorativos. Así, las evidencias cerámicas se entienden como un testimonio polisémico que puede aportar información muy relevante sobre cuestiones enormemente distintas referentes a los diferentes momentos del proceso de producción de un artefacto cerámico, a su uso, a las condiciones del entorno del si- tio arqueológico de donde proceden, a la estructura social de la producción, a las formas de transmisión del aprendizaje en una comunidad, etcétera.

Esta lenta pero inexorable evolución en la concepción del estudio de los productos cerámicos no es la primera que se produce en el ámbito del estudio y análisis de los distintos tipos de artefactos usados durante la prehistoria, donde cabe destacar, particularmente y por su precocidad respecto al resto, los planteamientos desarrollados en el estudio de la industria lítica tallada (ver Mora et al., 1992). Cabe remarcar, sin embargo, que en el ámbito de la investigación en torno a la cerámica Anna Shepard ya hizo en el año 1956 una propuesta ciertamente extensa en relación con el abanico de problemáticas que exponía y las metodologías posibles de aplicar en ese momento para cada una de las cuestiones que abordaba en su libro.

El número 23 de la revista Treballs d'Arqueologia está dedicado a recopilar un conjunto de trabajos centrados, cada uno de ellos, en uno u otro aspecto de las cuestiones que pueden plantearse en torno a la investigación de cualquier conjunto de cerámica prehistórica. No se ha pretendido desarrollar una exposición sistemática y exhaustiva de todas las cuestio-

1. Universitat Autònoma de Barcelona

2. Universitat de les Illes Balears 
nes de investigación que son posibles de abordar, aunque sí hemos querido poner de relieve la amplia diversidad de las cuestiones que se pueden llegar a plantear. En este sentido, los trabajos que se publican tienen una gran variedad de planteamientos teóricos, metodológicos, de estudios empíricos... No se ha buscado ni partir de un planteamiento teórico homogéneo en torno al cual se articularan las distintas aportaciones ni una discusión sobre el mayor o menor recorrido de cada una de las propuestas. Al contrario, la intención de esta publicación es que sean los lectores quienes, en todo caso, se posicionen desde sus respectivas posiciones teóricas y metodológicas, sobre el interés que pueda tener para ellos cada una de las propuestas y reflexionar hasta qué punto pueden creer conveniente incorporar alguna de estas o algunas de las preguntas que se plantean en sus investigaciones. De hecho, con esta publicación lo que queremos es contribuir a la renovación y al empuje de los estudios cerámicos sobre conjuntos de la prehistoria reciente peninsular, no porque, como queda claro con los trabajos que se presentan, no haya investigadores/ras que centren sus esfuerzo en este ámbito desde planteamientos muy diferentes, sino por que creemos imprescindible dar la mayor visibilidad a la variabilidad de posibilidades en la investigación sobre la cerámica prehistórica y poner sobre la mesa la conveniencia de explotar la máxima cantidad de información que sea posible a partir de las evidencias cerámicas, en tanto que entendemos que esto constituye una vía eficaz, tanto como cualquier otra, para profundizar en un gran número de cuestiones referentes a qué hicieron las comunidades del pasado, cómo lo crearon, para qué lo concibieron y en el marco de qué estructuras sociales, económicas, políticas, etcétera, lo forjaron.

El volumen que presentamos reúne trece artículos divididos en tres bloques.

El primer bloque está centrado en el estudio de la producción de cerámica. En él se incluyen cinco artículos que abordan diferentes aspectos del proceso de elaboración de la cerámica.

Xavier Clop incide en plantear la necesidad de precisar mejor determinados conceptos para poder abordar de manera más apropiada los estudios en torno a la caracterización de las materias primas utilizadas en la elaboración de cerámicas, y para que los resultados que se obtengan se incorporen de manera más sistemática a las discusiones más generales sobre las comunidades que realizaron estos productos analizados en cada caso.

Javier Cámara presenta una aproximación a la determinación de las técnicas de modelado a mano como una fértil vía para poder llegar a definir formas de hacer específicas a partir de la lectura rigurosa de las trazas que dejaron los gestos y las acciones desarrollados durante el proceso de formatización de los vasos.

Pilar Prieto muestra una metodología sistemática que hunde sus raíces en el concepto de chaîne opératoire y se inspira en los planteamientos de las escuelas de la antropología estructural francesa y de la arqueología contextual. Se centra en delimitar los rasgos decorativos que deben describirse para poder definir un patrón decorativo, cuya aplicación en el caso de Galicia desde el Neolítico Antiguo hasta el Bronce Final le ha permitido definir cuatro ciclos estilísticos o rupturas formales

Juan Jesús Padilla propone acercarnos al proceso de cocción de las cerámicas, desarrollando la idea de que el grado de espe- 
cialización en esta fase del proceso de producción no vendría marcado por la clase de estructura empleada o por los picos de temperatura alcanzados, sino por el dominio - o no- de determinados hábitos técnicos. Y como caso de estudio analiza las cerámicas documentadas en el yacimiento de Las Cogotas, desde la Edad del Bronce hasta el final de la Edad del Hierro.

Finalmente, Ignacio Clemente et al. desarrollan una cuestión hasta ahora poco valorada pero que sin duda es una línea de investigación a desarrollar en el futuro, como es la de la identificación, a partir de protocolos experimentales y análisis traceológicos, de los instrumentos de trabajo relacionados con la producción cerámica. Esta aproximación, además de mejorar nuestra visión y comprensión del proceso de producción cerámica en el pasado, permite plantear la cuestión de la posible identificación de los lugares donde efectivamente se desarrollaron esos procesos.

El segundo bloque está dedicado a trabajos que abordan cuestiones vinculadas a la etnoarqueología y la experimentación.

En el trabajo de Juan Salazar, Timothy Clack y Marcus Brittain se aborda, a partir de una investigación etnoarqueológica desarrollada en la zona baja del río Omo (Etiopía), la sustitución de una producción local de vasos cerámicos por otra foránea adquirida en el mercado, incorporando variables de carácter temporal, espacial y tecnológico, así como una metodología que combina la descripción tipológica con la obtención de testimonios orales. El análisis de todos estos elementos les permite plantear que el abandono de una tradición cerámica se manifiesta en múltiples esferas que superan el ámbito estrictamente tecnológico. En definitiva, se trata de un trabajo que aborda cuestiones vinculadas al papel social de la cerámica,

Jaume García Rosselló y Manuel Calvo Trías, partiendo de la concepción de que la investigación etnoarqueológica puede ser una estrategia experimental que permita obtener información de referencia válida para aplicar al estudio de las cerámicas de origen arqueológico, desarrollan una reflexión específica sobre el papel que han tenido las colecciones de cerámica etnográficas como elementos de referencia para la consolidación del método traceológico aplicado a la identificación de las técnicas de modelado, vía de estudio que progresivamente va adquieriendo mayor relevancia en la investigación arqueológica.

Sara Díaz-Bonilla trata del papel de la experimentación como una estrategia clave para entender las especifidades de las distintas fases que se desarrollan en los procesos de producción de cerámica durante la prehistoria, incluso de aquellas aparentemente más difíciles de estudiar de manera precisa, planteamiento que ilustran con la exposición de una propuesta de programa experimental destinado a la creación de un referente de trazas de tratamiento de las superficies que permite, también, establecer hipótesis fundamentadas sobre los posibles instrumentos de trabajos utilizados para hacer esos tratamientos.

En su segunda aportación a este bloque, Jaume García Rosselló y Manuel Calvo presentan otra propuesta de investigación centrada en la evaluación del potencial interpretativo de las macrotrazas de manufactura realizada a partir de un protocolo experimental sobre una colección de referencia. Se analiza tanto el comportamiento de las fracturas en las cerámicas como el de los resaltes y grietas 
generadas en la fractura trasversal en relación con las técnicas de manufactura utilizadas en la elaboración del vaso, con el objetivo de generar patrones extrapolables a las cerámicas prehistóricas.

Aixa Vidal aborda, a partir de distintos ejemplos etnográficos, diferentes cuestiones en torno a la variedad de situaciones de aprendizaje de las técnicas alfareras, y particularmente a las maneras y edades de inicio en esa actividad productiva, que se pueden registrar en sociedades no industrializadas, aplicando esas constataciones en la discusión de algunos ejemplos de cerámicas arqueológicas.

Finalmente, el tercer bloque lo hemos denominado cuestiones abiertas para recoger algunos ejemplos de temas y discusiones que afectan a nuestra concepción de las cerámicas prehistóricas y a su papel en las comunidades del pasado.

Isabel Rubio plantea la necesidad de reinterpretar el hallazgo de determinadas evidencias cerámicas en contextos en los que, según la perspectiva evolucionista que ha predominado en la construcción de muchos de los marcos interpretativos que prevalecen en la investigación arqueológica, no deberían encontrarse, constituyendo situaciones anómalas que, si se ponen en relación con informaciones aportadas desde la etnografía, deberían ser releídas y contextualizadas en el marco de determinadas prácticas y actividades desarrolladas por esas comunidades.

Finalmente, Eva Alarcón expone el estudio de recipientes cerámicos del sureste peninsular adscritos a la cultura del Argar desde la perspectiva de la arqueología feminista, de género y edad, centrán- dose en el análisis de contextos domésticos donde se materializaban tanto la vida cotidiana como determinadas prácticas funerarias.

Los editores de este volumen queremos agradecer a todos los autores y autoras de los trabajos que se recogen en el mismo haber aceptado formar parte de esta iniciativa y haber entendido perfectamente lo que propusimos en cada caso. Asimismo, les agradecemos el esfuerzo realizado al haber tenido que desarrollar sus trabajos en un plazo de tiempo relativamente corto.

También queremos agradecer a los revisores y las revisoras de los artículos, actores necesariamente anónimos en esta iniciativa, el trabajo que han realizado, que sin duda ha ayudado a mejorar el resultado final de los textos.

Finalmente, queremos agradecer al Servei de Publicacions de la Universitat Autònoma de Barcelona sus indicaciones a lo largo de todo el proceso de edición de este volumen y su trabajo, siempre contra reloj.

Esperamos que quien pueda leer los trabajos que se presentan a continuación acepte el reto de ampliar las discusiones tanto como sea necesario para que, entre todos y todas, mejoremos la capacidad de obtener información de los productos cerámicos realizados y utilizados por las comunidades prehistóricas y, así, contribuir a conocer mejor a los hombres y las mujeres que vivieron en uno u otro momento de la prehistoria reciente.

Bellaterra-Mallorca, noviembre de 2019 


\section{Referencias bibliográficas}

Alarcón García, E.; Padilla Fernández, J. J.; Arboledas Martínez, L.; Chapón, L. (eds.) (2018). «Algo más que galbos y cacharros. Etnoarqueología y experimentación cerámica». Menga, monográfico 04. Conjunto arqueológico Dólmenes de Antequera. Junta de Andalucía.

Albero Santacreu, D. (2014). Materiality, Techniques and Society in Pottery Production. Varsovia / Berlín: De Gruyter Open. <https://doi.org/10.2478/9783110410204>

Burnez-Lanotte, L. (2017). Matières à penser. Sélection et traitement des matières premières dans les productions potières du néolithique ancien. Séances de la Société Préhistorique Française, 11. París: Société Préhistorique Française.

García Rosselló, J.; Calvo Trias, M. (2013). Making pots: el modelado de la cerámica a mano y su potencial interpretativo. Oxford: BAR International Series 2540.

Hunt, A. M. W. (ed.) (2017). The Oxford Handbook of Archaeological Ceramic Analysis. Oxford University Press.

<https://doi.org/10.1093/oxfordhb/9780199681532.001.0001>

Mora, R.; Terradas, X.; Parpal, A.; Plana, C. (1992). «Tecnología y cadenas operativas líticas». Treballs d'Arqueologia, 1. Bellaterra: Universitat Autònoma de Barcelona.

Roux, V. (2016). Des céramiques et des hommes. Décoder les assemblages archéologiques. París (Francia): Presses Universitaires de Paris Ouest. (Versión en inglés publicada en 2019: Ceramics and Society. A Technological Approach to Archaeological Assemblages. Ed. Springer). <https://doi.org/10.1007/978-3-030-03973-8>

Shepard, A. O. (1956). Ceramics for the Archaeologist. 1. a ed. Washington: Carnegie Institution of Washington. 
